\title{
Datenschutz in Krisenzeiten oder Krise des Datenschutzes?
}

Sie haben mit diesem Heft der DuD soeben eine Zeitschrift gelesen, die bereits seit 1976 erscheint, nunmehr also bereits im 44 Jahr. Um das ein wenig besser einzuordnen: In Deutschland eröffnete Hessen 1970 mit dem ersten Datenschutzgesetz der Welt die Datenschutzgesetzgebung, im Jahr 1977 folgte dann der Bund mit der ersten Fassung des Bundesdatenschutzgesetzes (BDSG). Die Inhalte der DuD spiegeln also fast die gesamte Entwicklung des Datenschutzes und der zugehörigen Fachgesetzgebung in Deutschland wider.

Deutlich wird dabei: Der Datenschutz ist ein dynamisches Rechtsgebiet, dessen Anspruch ein Spiegelbild der immer schneller werdenden technologischen und soziologischen Entwicklungen ist. Zu Beginn sollte es ein „Gesetz zum Schutz vor Missbrauch personenbezogener Daten bei der Datenverarbeitung“ sein. Die erste „Krise“ des Datenschutzes war dem ursprünglichen Bundesdatenschutzgesetz immanent, denn die Datenverarbeitung wurde bereits seit 1980 zunehmend dezentral und sorgte so für die erste "Mängelliste“ des BDSG. In der Folge gab es eine Vielzahl von Interpretationen des Bundesdatenschutzgesetzes und die differenzierte Umsetzung in Landesdatenschutzgesetze. Das Ergebnis: In den Bundesländern entstanden teilweise unterschiedliche Rechtsnormen für gleiche „Tatbestände“, die für Wirtschaft und Verwaltung und viele Datenschutzbetroffene Unklarheit brachten und zudem zusätzliche Aufwände bedeuteten.

Ein weltumspannender Vorbehalt gegen den strengen Datenschutz entstand zudem durch die schnell wachsende breite Anwendung der Kryptographie. Bis heute beschreibt die „Kryptokontroverse" die gegensätzlichen Positionen der IT-Sicherheitsexperten versus der Strafverfolger und Geheimdienste zu verschlüsselten Daten und Informationen. Diese Krisensituation ist insbesondere aus Sicht des Datenschutzes ein permanentes Problem und hat in der Vergangenheit weltweit bereits zu unterschiedlichen gesetzlichen Regelungen geführt, die den Datenschutz teilweise eingeschränkt haben.

Auch durch die Corona-Pandemie ist so keine prinzipiell neue Situation entstanden, Krisen durchlebt der Datenschutz seit langem. Die im Rahmen des Homeschooling von einigen Datenschützern stark kritisierten eingesetzten Systeme und Technolo- gien gehören auf der anderen Seite aber seit langem zum Lebensalltag. Die „Hardliner“ im Datenschutz müssen sich daher fragen lassen, ob nicht gerade in Krisenzeiten an die Abwägung zwischen den -notwendigen und sinnvollen- Rechten des Einzelnen und der durch die Krise bedingten Anforderungen andere Maßstäbe anzulegen sind. Wenn der Datenschutz hier zum Hemmschuh beim Einsatz von ansonsten „Alltäglichem“ wird, ist dies den Betroffenen nicht zu vermitteln- und auf der Strecke bleibt langfristig nur eins: der Datenschutz.

Auch ist kritisch zu hinterfragen, ob und wie sich gesetzgeberische oder höchstrichterliche Vorgaben überhaupt noch in der aktuellen IT-Realität umsetzen lassen bzw. im Rechtsverständnis der Betroffenen plausibel sind. So sind das Recht auf ,,informationelle Selbstbestimmung “in seiner gegenwärtigen Lesart oder das vom Bundesverfassungsgericht in seinem Urteil vom 27. Februar 2008 statuierte „Grundrecht auf Gewährleistung der Vertraulichkeit und Integrität informationstechnischer Systeme" doch eher Postulate als in der modernen Internetwelt nachhaltig umsetzbare Ziele. Ein jüngstes Beispiel für diffuse Interpretationen bietet auch das ,Judgement of the Court of Justice of the European Union in Case C-311/18 - Data Protection Commissioner v Facebook Ireland Ltd and Maximilian Schrems" vom 16. Juli 20201, mit dem bisherige „Vertrauensanker“ großer Teile der Internetgemeinschaft außer Kraft gesetzt wurden. So scheint eine neue Phase der Verunsicherung unvermeidlich.

Die (deutschen) Aufsichtsbehörden müssen sich daher in ihrem Vorgehen mit den Experten im Europäischen Datenschutzausschuss abstimmen und zukünftig auch zu spezifischeren Fragestellungen beraten. Dabei darf allerdings die bestehende Wirklichkeit nicht verdrängt werden - zumindest dann nicht, wenn der Datenschutz langfristig Akzeptanz und damit auch Durchsetzungskraft behalten soll. Denn nur wenn die Anforderungen des Datenschutzes - insbesondere bei den „Betroffenen“ nicht nur auf Verwunderung und Ablehnung stoßen, lässt sich das eigentliche Ziel des Datenschutzes verwirklichen: der Schutz des Einzelnen vor Missbrauch seiner personenbezogenen Daten.

\footnotetext{
1 Siehe auch Dokumentation in diesem Heft
} 